\title{
Study the effect of exercise on systolic pulmonary artery pressure in healthy subjects
}

\author{
Masoud Seyedian, Farzane Ahmadi", Atefeh Lalvand, Mohammad Nourizadeh \\ Cardivascular Disease Research Center, Ahvaz Joundishapour University, Ahvaz, Iran; \\ *Corresponding Author: AhmadiTHC@yahoo.com
}

Received 2 December 2011; revised 9 January 2012; accepted 18 January 2012

\begin{abstract}
Introduction: As no data are available concerning the cut-off value defining abnormal pulmonary artery systolic pressure (PASP) response in subjects of various ages, the aim of this study is to assess physiological PASP response to exercise in healthy individuals of various ages. Material and methods: One hundred and twenty three healthy volunteers, aged $\mathbf{3 0}$ to $\mathbf{7 0}$ years, underwent Doppler echocardiographic measurements at rest and after treadmill exercise test. Pulmonary artery systolic pressure was estimated at rest, and immediately after peak exercise using Bernoulli formula (four times tricuspid valve regurgitation velocity squared adding an estimated right atrial pressure). Results: Lower and upper limits of PASP during rest was 7 and $28 \mathrm{mmHg}$ and after peak exercise was 14 and 48 $\mathrm{mmHg}$ respectively. After exercise, PASP increased from rest $(14 \pm 4 \mathrm{mmHg})$ to peak $(25 \pm 7$ $\mathrm{mmHg}$ ). Pulmonary artery systolic pressure during rest and peak exercise, increased with age, but has no correlation with body mass index or gender. Conclusion: Pulmonary artery systolic pressure at peak exercise can frequently reach values $\geq 30 \mathrm{mmHg}$ in healthy individuals with good exercise capacity, especially in elderly individuals, which goes beyond pathologic definitions of pulmonary hypertension.
\end{abstract}

Keywords: Pulmonary Arterial Hypertension; Pulmonary Arterial Systolic Pressure; Exercise; Doppler Echocardiography

\section{INTRODUCTION}

Pulmonary arterial hypertension (PAH) is known to diminish exercise capacity by decreasing right ventricular performance, pulmonary blood flow, and oxygen delivery to peripheral musculature during exercise [1]. Al- though a number of studies showed that pulmonary artery systolic pressure (PASP) slightly increase with exercise in healthy volunteers and reaches to a value of 35 or $40 \mathrm{mmHg}$, [2-5] some authors believe that because these findings are based on evaluation of young healthy subjects, PASP at exercise may reaches to higher value in healthy older people [1]. As it is not a definition for lower and upper limits of normal PASP fitting to all individuals and all exercise levels, and according to introduction of Doppler quantification of tricuspid regurgitation peak velocity as a reliable instrument to assess PASP at rest and exercise, we sought to explore the range of pulmonary artery systolic pressure at rest and with exercise in healthy individuals of various ages.

\section{MATERIAL AND METHODS}

All of the subjects in this cross sectional study were healthy volunteers, without cardiopulmonary disorders, aged 30 to 70 years. At the beginning of study, subjects were weighted and their height was measured. So their body mass index (BMI) was deliberated. Regarding the BMI, subjects divided into four groups: lean $(\mathrm{BMI}<20)$, Normal (BMI 20 - 25), Overweight (BMI 25 - 30), and Obese (BMI > 30) volunteers. Electrocardiogram and chest $\mathrm{x}$-ray of all subjects were recorded and their serum electrolytes were measured. Subjects were excluded from the study regarding to have history of chronic cardiopulmonary disorders, signs or symptoms of cardiopulmonary disease, abnormal ECG (including old MI, AV block), abnormal chest $\mathrm{x}$-ray, electrolyte imbalance, and mental or physical deficit that make the subject unable to do the exercise test. Also subjects were excluded if they had systolic blood pressure $>200 \mathrm{mmHg}$ or diastolic blood pressure $>110 \mathrm{mmHg}$ Just before the echocardiographic assessments. All of the subjects underwent Trans thoracic 2 dimension and color Doppler echocardiography (using the GE Vivid 3 Export GE 2007) at rest and If any abnormality (including right ventricle dilatation, left ventricle diastolic dysfunction, more than trivial valvular insufficiency, pulmonary arterial hyper-tension with 
sever tricuspid regurgitation) was found, subject was excluded. Also if ischemic changes were observed during exercise test, subject was disqualified for this study.

In this way, 123 healthy volunteers enrolled in to this study. Continues wave Doppler echocardiography in multiple views was carried out at rest and in supine position to find the peak tricuspid regurgitation velocity. Right atrial pressure (RAP) was estimated regarding the diameter of inferior vena cava, tricuspid regurgitation, and right atrium size (Table 2).

Using the Bernoulli formula (four times tricuspid valve regurgitation velocity squared adding an estimated right atrial pressure) the PASP of subjects at rest were estimated.

Then each healthy subject, carried out the treadmill exercise test and after they reach the target heart rate (eighty five percent of (220-age) for each subject) the treadmill exercise was stopped and subjects underwent Doppler echocardiography immediately and PASP of each subject was estimated again. Each experiment performed one time, then data recorded by physician in the questionnaires.

Data are presented as the mean \pm standard deviation (SD) or percentage as appropriate. Chi-square test or Fisher's exact test was used for comparisons of dichotomous data. Pearson coefficient was used for correlation of quantitative data. Assessment of mean between the 2 groups was performed using the one sample independent $\mathrm{t}$ test. A p value less than 0.05 was considered significant. all data were analyzed by SPSS 17 software.

The Research Ethics Committee of the Ahwaz Joundishapour University of Medical Sciences approved this study at $2010 / 12 / 5$ and with the identification number of $2726 / \mathrm{d} / 20 / 8 / \mathrm{p}$.

\section{RESULTS}

One hundred and twenty three healthy volunteers, 58 men and 65 women (mean age $47 \pm 11$ years), with at least 20 subject in each range of age (30 - 40, 40 - 50, 50 - 60, and 60 - 70) enrolled into this study. Mean BMI of subjects was $25 \pm 4 \mathrm{~m} / \mathrm{kg}^{2}$, showing no differences between two genders $(\mathrm{p}=0.68)$. There were seventy lean, 48 normal, 48 overweight, and 10 obese subjects (Table 1).

Mean PASP at rest was $14.5 \pm 4.5 \mathrm{mmHg}$ (ranging from 7 to $28 \mathrm{mmHg}$ ) among the subjects with no differrences between two genders or various BMI. PASP at rest increased simultaneously with age $\left(r^{2}=224, p<\right.$ 001). After peak exercise PASP increased to $25.5 \pm 7$ $\mathrm{mmHg}$ (ranging from 14 to $48 \mathrm{mmHg}$ ). It shows no differences between 2 genders or various BMI, but increased with age concurrently $\left(\mathrm{r}^{2}=356, \mathrm{p}<001\right)$.

PASP difference before and after exercise test had a positive linear correlation with age $\left(r^{2}=21, p<001\right)$ and also PASP at rest $\left(r^{2}=05, p=015\right)$, but not with gender or BMI (Figure 1).

As you can see in Figure 1, elevated PASP before exercise leads to more elevation of PASP after exercise.

\section{DISCUSSION}

Previous invasive studies described mild increases in pulmonary pressure with exercise in the normal population [6]. Dokainish et al. assumed that PASP may reaches the value of more than $40 \mathrm{mmHg}$ in the healthy elderly people ( $>90$ years) [7]. Mahjoub et al. showed that PASP more than $60 \mathrm{mmHg}$ in old peoples ( $>60$ years old) could be considered normal [1]. In our study that included healthy men and women of various ages, PASP at peak exercise reach values $\geq 30 \mathrm{mmHg}$ (like Kovacs study). As age increase, pulmonary blood flow decrease, mean pulmonary pressure increase, and an increase occur in pulmonary resistance, supposed that related to reduced compliance of the pulmonary bed [8]. So as suggested by Bossone et al., one should put workload and age with PASP response together to decide if an increase in PASP is a pathologic occurrence or not [2]. Ha et al. showed

Table 1. Patients characteristics.

\begin{tabular}{ccccccc}
\hline & & & Number of patients & Male & Female & p value \\
\cline { 3 - 6 } & & & 123 & $58(47.2)$ & $65(52.8)$ & 0.39 \\
\hline \multirow{2}{*}{ AGE } & MEAtients & $47.3 \pm 11$ (years) & $46.78 \pm 110.4$ & $47.7 \pm 10.66$ & 0.619 \\
BMI & MEAN & $25.2 \pm 3.95\left(\mathrm{~kg} / \mathrm{m}^{2}\right)$ & $25.83 \pm 4.05$ & $25.09 \pm 3.88$ & 0.689 \\
\hline
\end{tabular}

Table 2. Estimation of right atrial pressure regarding the diameter of inferior vena cava, tricuspid regurgitation, and right atrium size.

\begin{tabular}{|c|c|c|c|c|}
\hline Inferior vena cava diameter & Tricuspid regurgitation velocity & Tricuspid regurgitation & Right atrium size & $\begin{array}{l}\text { Estimated right atrial } \\
\text { pressure }\end{array}$ \\
\hline Normal & Equal to or less than $2.5 \mathrm{~m} / \mathrm{s}$ & None or mild & Normal & $5 \mathrm{mmHg}$ \\
\hline Dilated & 2.6 to $4 \mathrm{~m} / \mathrm{s}$ & Moderate & $\uparrow$ & $10 \mathrm{mmHg}$ \\
\hline Dilated, without respirator collapse & More than $4 \mathrm{~m} / \mathrm{s}$ & Severe & $\uparrow \uparrow$ & $15 \mathrm{mmHg}$ \\
\hline
\end{tabular}




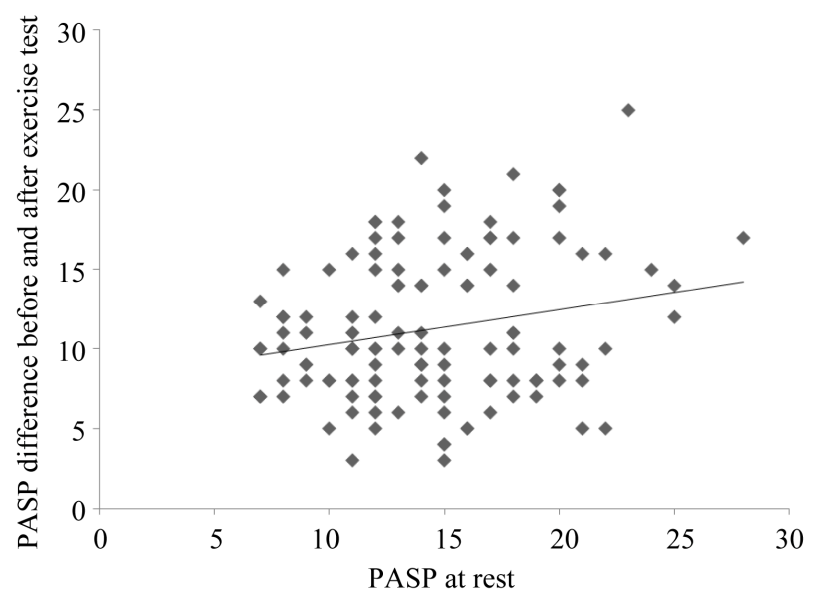

Figure 1. Correlation of PASP difference before and after exercise and PASP at rest.

that resting TR velocity, E/E', age and gender are the strongest predictors of PASP during exercise. They also proposed a value of $50 \mathrm{mmHg}$ for a workload of $50 \mathrm{~W}$ to define pulmonary hypertension at exercise [9].

In our study, there were no correlations between gender, BMI and PASP at rest or PASP at peak exercise (p. 0.05). The data of our review, like Kovacs research, do not support the current threshold of PASP during exercise and it was shown that, exercise PASP is age related and frequently exceed $30 \mathrm{mmhg}$, especially in elderly individuals, which make it difficult to define normal PASP during exercise [10,11]. PASP after exercise had correlation with age and PASP before exercise (Figure 1) and subjects with more PASP before exercise had more elevation of PASP after exercise. In a large study on 3790 echocardiographically normal subjects by McQuillan et al. results showed that PASP was associated with age, BMI, sex, wall thickness, and ejection fraction. It is a fact that our sample size is smaller than McQuillan study, therefore our study suffered from potential selection bias. Also it should be noted that in McQuillan study, right atrial pressure considered as a constant value of 10 $\mathrm{mmHg}$, but we estimate it as 5,10 , or $15 \mathrm{mmHg}$ regarding the diameter of inferior vena cava, tricuspid regurgitation, and right atrium size.

\section{STUDY LIMITATIONS}

Invasive measurement of PASP at rest and during exercise was not performed to match up to with our echocardiographic data. Although the assessment of PASP by Doppler echocardiography is reliable at rest, the accuracy of this method is not fully established during exercise. We just evaluated the PASP at rest and peak exercise and did not perform our assessment during treadmill exercise test. Thus raise of PASP in early stage of exercise test in individuals can be suggestive for pulmonary hypertension. We did not use any contrast (such as air and saline) to define the tricuspid regurgitation velocity better.

\section{CONCLUSION}

Pulmonary artery systolic pressure at peak exercise can reach values $\geq 40 \mathrm{mmHg}$ in healthy individuals, especially older peoples. Further studies in large populations are required to determine the abnormal range of PASP at various level of exercise, and also compare the result of echocardiography and invasive methods during exercise.

\section{REFERENCES}

[1] Mahjoub, H., Levy, F., Cassol, M., Meimoun, P., Peltier, M., Rusinaru, D., et al. (2009) Effects of age on pulmonary artery systolic pressure at rest and during exercise in normal adults. European Journal of Echocardiography, 10, 635-640. doi:10.1093/ejechocard/jep024

[2] Bossone, E., Rubenfire, M., Bach, D.S. Ricciardi, M. and Armstrong, W.F. (1999) Range of tricuspid regurgitation velocity at rest and during exercise in normal adult men: implications for the diagnosis of pulmonary hypertension. Journal of the American College of Cardiology, 33, 16621666. doi:10.1016/S0735-1097(99)00055-8

[3] Janosi, A., Apor, P., Hankoczy, J. and Kadar, A. (1988) Pulmonary artery pressure and oxygen consumption measurement during supine bicycle exercise. Chest, 93, 419-421. doi:10.1378/chest.93.2.419

[4] Collins, N., Bastian, B., Quiqueree, L., Jones, C., Morgan, R. and Reeves, G. (2006) Abnormal pulmonary vascular responses in patients registered with a systemic autoimmunity database: Pulmonary hypertension assessment and screening evaluation using stress echocardiography (PHASE-I). European Journal of Echocardiography, 7, 439-446. doi:10.1016/j.euje.2005.12.002

[5] Grünig, E., Janssen, B., Mereles, D., Barth, U., Borst, M.M., Vogt, I.R., et al. (2000) Abnormal pulmonary artery pressure response in asymptomatic carriers of primary pulmonary hypertension gene. Circulation, 102, 1145-1150.

[6] West, J.B. (1998) Left ventricular filling pressures during exercise: A cardiological blind spot? Chest, 113, 1695-1697. doi:10.1378/chest.113.6.1695

[7] Dokainish, H., Gin, K., Lee, P.K. and Jue, J. (2003) Left ventricular filling patterns and pulmonary artery pressures in patients aged 90 to 100 years with normal echocardiography results. Journal of the American Society of Echocardiography, 16, 664-669. doi:10.1016/S0894-7317(03)00227-X

[8] Wagner, P.D., Gale, G.E., Moon, R.E., Torre-Bueno, J.R., Stolp, B.W. and Saltzman, H.A. (1986) Pulmonary gas exchange in humans exercising at sea level and simulated altitude. Journal of Applied Physiology, 61, 260-270.

[9] Ha, J.W., Choi, D., Park, S., Shim, C.Y., Kim, J.M., Moon, S.H., et al. (2009) Determinants of exercise-induced pul- 
monary hypertension in patients with normal left ventricular ejection fraction. Heart, 95, 490-494.

doi:10.1136/hrt.2007.139295

[10] McQuillan, B.M., Picard, M.H., Leavitt, M. and Weyman, A.E. (2001) Clinical correlates and reference intervals for pulmonary artery systolic pressure among echocardiographically normal subjects. Circulation, 104, 2797-2802. doi:10.1161/hc4801.100076

[11] Kovacs, G., Berghold, A., Shiedel, S. and Olschewseki, H. (2009) Pulmonary artery pressure during rest and exercise in healthy subjects: A systematic review. European Respiratory Journal, 34, 888-894. doi:10.1183/09031936.00145608 